\title{
SM Otolaryngology A De Novo from Mutational Analysis in PAX3 in Hearing Loss Children Caused Waardenburg Syndrome Type 1 at Children Hospital-1 in Ho Chi Minh City, Vietnam
}

Nguyen Dinh Pham ${ }^{1}$, Dung Huu Nguyen ${ }^{2}$, Tran Huyen Lam², Kieu Tho Nguyen², Chuong Dinh Nguyen ${ }^{2}$, Hoang Nguyen ${ }^{3 *}$, Joseph P Kitzmiller ${ }^{3}$, Vu Anh Hoang ${ }^{4}$, Thuong Chi Bui ${ }^{5}$ and Toan Thanh Pham ${ }^{6}$

${ }^{1}$ Department of Otolaryngology, Children Hospital 1, HCM City, Vietnam

${ }^{2}$ Department of Otolaryngology, The University of Medicine and Pharmacy at HCM City, Vietnam

${ }^{3}$ Department of Biological Chemistry and Pharmacology, The Ohio State University, College of Medicine, USA

${ }^{4}$ The University of Medicine and Pharmacy at HCM City, The Center for Molecular Biomedicine, Vietnam ${ }^{5}$ The University of Medicine and Pharmacy at HCM City, Department of Obstetrics/Gynecology, Vietnam

${ }^{6}$ University of Natural Science, Vietnam

\section{Article Information}

Received date: Jul 28, 2017

Accepted date: Aug 21, 2017

Published date: Aug 25, 2017

\section{*Corresponding author}

Hoang Nguyen, The Ohio State University, College of Medicine, Department of Biological Chemistry and Pharmacology, Columbus, Ohio, USA, Email: Hoang.Nguyen@osumc.edu

Distributed under Creative Commons CC-BY 4.0

Keywords PAX3 gene; Congenital sensorineural hearing loss; c.955delC (Gln319fsX380); c.667C>T (Arg223Stop); Waardenburg Syndrome type 1; Children in Hospital-1; Ho Chi Minh City

Article DOI 10.36876/smotol.1010

\section{OPEN ACCESS}

ISSN: 2574-2418

\section{Abstract}

Purpose: To detect a de novo paired box gene 3 (PAX3) mutations, mutational analysis, and associated phenotypes in the Vietnamese Children patients with Waardenburg syndrome type I (WS1). We conduct this study to provide initial information about types, rates, and influences of this particular PAX3 mutations gene in the Vietnamese patients.

Patients and Methods: Three unrelated patients with suspected WS1 were selected from our Genomic extraction DNA, probe and sequence the primers, PCR technique. PAX3 were amplified by polymerase chain reaction $(\mathrm{PCR})$, and then amplicons $\mathrm{PCR}$ were analyzed by cycle sequencing. Variations were detected, documented and recognized as "de novo" mutation. We also compared the severity of hearing impairment, phenotypically variations among these individual affected patients.

Results: Number variations of mutations in PAX3 were detected in three patients, respectively: c.955delC (GIn319fsX380), c.667C>\% (Arg223Stop). One mutation proved to be de novo as their parents did not carry the mutations, and had never been found in any previous study in Vietnam. All three patients with PAX3 mutations had different iris color, fundi between their two eyes, dystopia canthorum and profound hearing loss. We report the phenotypic expression of WS1 in these three patients and explore the implications for possible genotypephenotype correlations. Sensorineural hearing loss was present in $80 \%$ of affected individuals, and spectrum of hearing loss with very high frequency.

Conclusion: De novo mutation in PAX3 has not been reported in Vietnam before. Finally, our finding has detected deletion mutation c.667>T (p.Arg223X) in PAX3 identified in three children individual with WS1. Our analyses indicated that these mutations might constitute a pathogenic-genetic-hearing loss associated with WS1. It is very clinical importance as such patients may be misdiagnosed as congenital hearing loss since it is uncommon in Southern Vietnamese population.

\section{Introduction}

Waardenburg Syndrome (WS) is named after Dutch ophthalmologist Petrus Johannes Waardenburg (1886-1979), who described the syndrome in detail in 1951. It is the most common type of autosomal dominant syndromic hearing loss. It consists of variable degrees of hearing loss and pigmentary abnormalities of skin, hair (white forelock), and eye (Heterochromia iridis). Ten subtypes and four types are recognized: WS1, WS2, WS3, and WS4. Phenotypic among these types are difference: WS1 is characterized by presence of dystopia canthorum while WS2 is characterized by its present. In WS3, upper-limb abnormalities are present, and finally in WS4, Hirschsprung disease is present. Overall, the syndrome affects approximately 1 in 45000 people [1].

WS1 is a pigmentary-auditory disorder comprising congenital sensorineural hearing loss and other pigmentary disturbances. The hearing loss in WS1, observed in approximately 70\% of affected individuals. It is congenital, typically non-progressive, either unilateral or bilateral, and sensorineural [2]. Mutations in the paired box gene 3 (PAX3) have been identified to be responsible for WS1. Identification of a heterozygous PAX3 pathogenic variant by molecular genetic testing establishes the diagnosis if clinical features are inconclusive [3]. PAX3 is a gene that belongs to the paired box (PAX) family of 
transcription factors. This gene was formerly known as splotch. PAX3 has been identified with ear, eye and facial development. It is expressed in early embryonic phases in pigment system, and in dermatomyotome of paraxial mesoderm which it helps to demarcate. PAX3 encodes a member of the mammalian PAX family of transcription factors, which contains two highly conserved domains for DNA binding, paired box domain and paired-type homeodomain [4]. PAX3 is active in neural crest cells. In conjunction with MSX1, PAX3 guides the expression of the molecule; this allows neural crest cell plays very important role on organ development. Thus, mutating of PAX3 results neural crest cell can't complete their function as formation development role, secondary to impair hearing loss [5]. This mutation results in replacing the amino acid asparagines with lysine, inhibiting the PAX3 protein from binding to the necessary DNA. Alternative splicing of PAX3 results in several different length transcripts, of which the longest transcript contains 10 exons, and consequent proteins with distinct carboxyl termini. Heterozygous mutations in PAX3 have been reported in familial and sporadic WS1, while heterozygous or homozygous mutations have been detected in patients with WS3. Numerous mutations have been identified in Caucasians, minimal cases have been determined in Asian population. However, WS1 patient with PAX3 mutations in Vietnam have not been studied or reported.

In this study, we conducted detailed, thorough clinical and genetic analysis of several Vietnamese children individuals with WS1-afflicted. Several mutations in PAX3 were identified in three individual children from unrelated families with WS1. A de novo heterozygous deletion mutation c.667C $>\mathrm{T}$ (p.Arg223X) was also detected in the patient. All patients with mutations presented dystopia cathorum, different colors of irises, and profound hearing loss. Since pathogenic variant has been identified in an affected children, prenatal testing option from a clinical laboratory that offers either testing for this gene or custom prenatal testing. Molecular genetic testing of relatives at risk allows for early screening of those at risk for hearing loss.

\section{Subjective and Methodology}

\section{Patients}

Three unrelated patients were referred from our Pediatric and Genetic Ophthalmology Department at Nhi Dong I Children Hospital, HCM City Vietnam. There are several highlighted clinical features, but they are very inconclusive. In addition to profound hearing loss, one patient has hypoplastic blue irides, other had delayed speech pathology. No other clinical symptoms have met major, minor criteria to establish the diagnosis of WS1, thus, diagnosis of WS1 aren't confirmed to two major criteria or one major plus two minor criteria aren't met. Therefore, we tried to identify of a heterozygous pathogenic variant in PAX3 by molecular genetic testing, and result is positive. Informed consent conforming to Center for Molecular Biomedicine at The University of Medicine and Pharmacy at HCM City, HCM City, Vietnam; all participants received detailed ophthalmological and otolaryngological examinations performed by ophthalmologist, and otolaryngologist. All patients signed the consents and followed the IRB Review of Vietnam guideline/protocols.

\section{Research methodology}

We are reporting 3 cases in each three individual patients with congenital sensorineural hearing loss with features characteristic for
WS1, and PAX3 gene have been extracted, analyzed for further evaluation of de novo gene definition.

Case presentation 1: The 12 years old boy was referred from Ophthalmology Department for genetic counseling and further evaluation due to congenital hearing loss. The proband is the only child of non-consanguineous parents. He was born at term after an uneventful pregnancy. His birth weight was 3200 gram; length $53 \mathrm{~cm}$. Apgar score was 10 . His early psychomotor development was normal. Due to his profound hearing loss, he attended a school for children with hearing impairment. At the age of 10 , he was hospitalized due to minor car accident has been hit by motorcycle in Ho Chi Minh City. Phenotype examination at genetic counseling center revealed the presence of wide set eyes of hypoplastic blue irides, and dystopia canthorum. He did not present white forelock characteristic for WS. Intellectual development was normal. Both parents were phenotypically normal and neither of them presented any features suggestive of WS.

Case presentation 2: The 8 years old boy was also referred for genetic counseling due to hypoplastic irides in addition to congenital hearing loss. The proband is the first child of non-consanguineous parents. His younger sister- 5 years old, who has the same problem. They were born at term after an uneventful pregnancy. Their early psychomotor development was normal, without any notable delay up to 2 years of age. Later both presented speech impairment due to hearing problems, noted at the age of 3. Both attended a school for children with hearing impairment. Both parents were congenital hearing impairment, but lacking hypoplastic irides. Interesting, their first auntie was also congenital hearing impairment, and noted features of hypoplastic irides suggestive of WS. Due to economic unstablility, living with social inequality; both parents and their first auntie have relocated to different regions of the country, thus we can't obtain their DNA sample for gene mutations analysis.

\section{Methodology}

\section{DNA genomic extraction}

For molecular testing, DNA was extracted from peripheral blood (venous leukocytes) samples using standard nonorganic protocols. Bloods were stored with EDTA agent, and transported to Molecular Genetic Institute at HCMU School of Medicine and Pharmacy. Genomic DNA was extracted from $200 \mu \mathrm{L}$ peripheral blood by using QIAamp DNA kit (Quiagen, USA), followed by manufacturing instruction.

\section{Probe and sequencing primers}

To detect mutations in exon 3 of the PAX3 gene, we used the strategy of directly sequencing PCR-amplified DNA of an affected individual from each patient. Oligonucleotide primers $\mathrm{CH} 2-1$ and $\mathrm{Ch} 2-3$ were used to amplify human PAX3 exon3. The amplification product from $50 \mathrm{ml}$ reaction was purified by membrane. To amplify human PAX3, the first strand CDNA was used as a template for a first round of amplification to amplify from exon 2 to the last 18 bases of coding sequence with oligonucleotide primers HUP2-4 and PAX3-1. Three additional nested amplifications were performed by using the product of each preceding reaction as a template. The primer pairs used were HUP2-5 (5' end of exon 3) with PAX3-1 (last 18 bases of coding sequence), HUP2-5 (5' end of exon 3) with PAX3-13 (64 bases upstream from stop codon). PAX3 is code as "NG_011632" on GenBank. Pairs of primer were designed based on primer3 http://bioinfo.ut.ee/primer3-0.4.0/). The primers for 
Table 1: Reaction of PCR and temperature/interval on gen PAX3 gene.

\begin{tabular}{|c|c|c|c|c|c|}
\hline \multicolumn{2}{|c|}{ Reactions } & \multicolumn{4}{|c|}{ Temperature and Interval of probe PCR } \\
\hline PCR conducted & Volume $(\mu \mathrm{l})$ & Periods & Temp & Times & Interval \\
\hline $2.5 \mathrm{mM}$ dNTP & 1.5 & Denatured & $98^{\circ} \mathrm{C}$ & $10 \mathrm{sec}$ & \multirow{3}{*}{40} \\
\hline $10 \mu \mathrm{M}$ primer $\left(\mathrm{F}_{2}\right.$ and $\left.\mathrm{R}_{2}\right)$ & 1.5 & Binding & $60^{\circ} \mathrm{C}$ & $20 \mathrm{sec}$ & \\
\hline $\mathrm{H}_{2} \mathrm{O}$ & 8.4 & Prolonged & $72^{\circ} \mathrm{C}$ & $40 \mathrm{sec}$ & \\
\hline Takara HS Taq & 0.1 & & $72^{\circ} \mathrm{C}$ & $3 \min$ & \\
\hline gDNA & 2.0 & & & & \\
\hline Total Volume & 25 & & & & \\
\hline
\end{tabular}

exon F2 were as follows: (5'-GTCCTAGCTAGTGATTCCTG-3') and exon R2 (5'-GTTGCCTCATCCCTCTCATG-3'), a 50-bp product was obtained after amplification. The amplicons from individual exon were purified and analyzed by cycle sequencing with Integrated DNA Technologies (USA). Sequencing results from patients were imported into NCBI Human Genome Database for comparing and filtering any de novo mutation gene. Each variation was confirmed by bidirectional sequencing.

\section{PCR technique}

PCR of the PAX3 exons was performed in a total volume of $25 \mu \mathrm{L}$ containing 50-100ng of genomic DNA, $500 \mathrm{nM}$ each of the forward and reverse primers, $500 \mu \mathrm{M}$ dNTPs, $(250 \mu \mathrm{M}$ for each and $1.25 \mathrm{U}$ LA Taq DNA polymerase with GC buffer I from TAKARA (Tokyo, Japan). The amplification consisted of an initial denaturation stage at $98^{\circ} \mathrm{C}$ for $3 \mathrm{~min}$, followed by 40 cycles consisting of denaturation at $98^{\circ} \mathrm{C}$ for $30 \mathrm{~s}$, annealing for $15 \mathrm{~s}$ at $60^{\circ} \mathrm{C}$, and extension at $72^{\circ} \mathrm{C}$ for 1 minute, with an extension step performed at $72^{\circ} \mathrm{C}$ for $5 \mathrm{~min}$ (Table 1 ).

PCR product were dyed in agarose gel $1.5 \%$ with ethidium bromide and observed by camera photographer Geldoc-It (UVP, USA). Finally, PCR was purified, buffered by illustra ${ }^{\mathrm{TM}} \mathrm{GFX}^{\mathrm{TM}} \mathrm{PCR}$ DNA and Gel Band Purification Kit (GE Healthcare, England) based on the manufacturing instruction.

\section{Perform DNA sequencing}

PCR fragments were sequenced using the forward and/or reverse primers and ABI BigDye Terminator v3.1 Cycle Sequencing Kit (Applied Biosystems, Inc., Foster City, CA) and ABI 3130 Genetic Analyzer. The product is then precipitated with ethanol, dissolved in $\mathrm{Hi}-\mathrm{Di}$ formamide, denatured at $95^{\circ} \mathrm{C}$ in 2 minutes before the sudden cooling. DNA sequence later read by ABI 3130 Genetic Analyzer, With POP-7 polymer and $80 \mathrm{~cm}$ capillary (Applied Biosystems, USA). Results are plugged in and analyzed by CLC Main Workbench software.

\section{Statistical analysis}

All experiment results were analyzed by Excel and IBM SPSS v.20.0 with 95\% Confident Interval (CI)

\section{Results and Discussion}

\section{General characteristic of experiments}

Inclusion criteria were: 1) congenital; 2) clinical presentation: have one sign or symptoms belongs to major or minor criteria 3 ) type of hearing loss: sensorineural; 4) prognosis: stable/progressive HL; 5) any ear affected; 6) sporadic or familial case in his/her family

Exclusion criteria were: 1) non-syndromic hearing loss; and 2) congenital hearing loss caused by infections. The period of study took place between April, 2015 to September, 2016.

\section{Variation detection}

In the 3 patients, numerous heterozygous mutations in PAX3 were detected, including c.955delC (Gln319fsX380), c.567_586+17del (p.Asp189_Gln505delinsGluGlyGlyAlaLeuAlaGly), c.456_459dupTTCC (p.Ile154PhefsX162), c.795_800 delCTGGTT(p. Trp266_Phe267del), c.799T>A (p.Phe267Ile), and c.667C $>$ T (p.Arg223X). The c.567_586+17del mutation was identified in a baby from Family A. Direct sequencing revealed a heterozygous variation involving multiple nucleotides in exon 4 region. Cloning sequencing revealed a $37 \mathrm{bp}$ deletion exon 4 and intron 4 . A new splice site is predicted to be created downstream. The encoded protein would be truncated. The c.456_459dupTTCC and c.795_800delCTGGTT mutations were only present in the probands. Mutation c.667C>T (p.Arg223X) is proved to be de novo as their parents did not carry the mutations, suggesting that natural occurring new mutations in PAX3 of the Vietnamese population is not uncommon. Based on available information, de novo mutations in PAX3 have rarely been mentioned before. C.667C $>\mathrm{T}$ (p.Arg223X), are predicted to encode premature truncated proteins affecting the paired-type homeodomain.

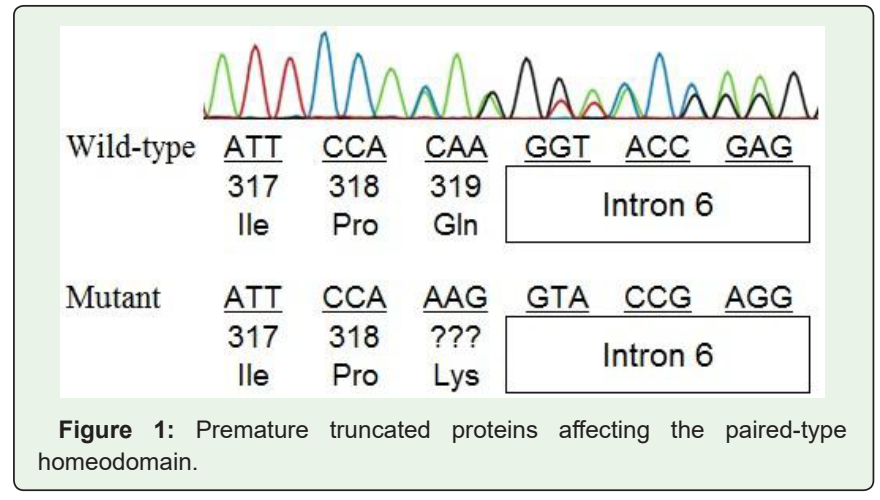




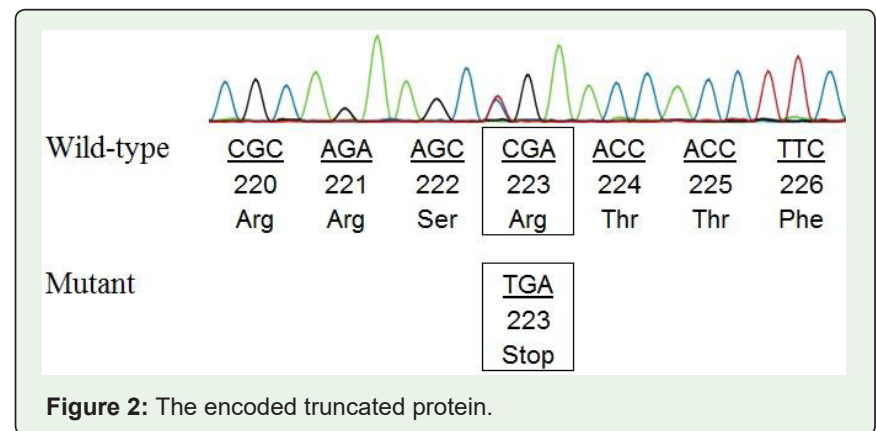

\section{Conclusion}

In summary, we described a de novo heterozygous deletion mutation in 2 Vietnamese families with WS1. Our analyses indicated that these mutations are highly associated with congenital hearing loss. This will be the root solution to confirm diagnosis with better treatment.

\section{Acknowledgements}

The authors are grateful to all medical staffs, statistician for assistance with data analysis. The authors are grateful for the assistance and expertise in laboratory analysis of the laboratory staff of Department of Otolaryngology, Children Hospital 1, HCM City, Vietnam; The University of Natural Science, Department of Biochemistry, HCM City, Vietnam; The Center of Molecular and Genetic Medicine, The Department of Otolaryngology, and the
Department of Obstetrics/Gynecology, of The University of Medicine and Pharmacy at HCM City, HCM City, Vietnam. Special thanks to scientists and professors at The Ohio State University, College of Medicine, Department of Biological Chemistry and Pharmacology, Columbus, Ohio, USA for re-evaluate, recommend and validate important basic intellectual concepts correlate to clinical. The authors are also thankful for the oversight of all aspects of this research but the Internal Review Boards (IRB) in Vietnam, as well as Department of Public Health, Board of Medicine in Vietnam.

\section{References}

1. Baldwin CT, Hoth CF, Macina RA, Milunsky A. Mutations in PAX3 that cause Waardenburg syndrome type I: ten new mutations and review of the literature. Am J Med Genet 1995; 58: 115-122.

2. Markova TG, Megrelishvilli SM, Shevtsov SP, Shvarts EI. Clinical and molecular genetic investigation of Waardenburg syndrome type 1. Vestn Otorinolaringol 2003; 1: 17-19.

3. Pierpont JW, Doolan LD, Amann K, Snead GR, Erickson RP. A single base pair substitution within the paired box of PAX3 in an individual with Waardenburg syndrome type 1 (WS1). Hum Mutat 1994; 4: 227-228.

4. Tassabehji M, Read AP, Newton VE, Harris R, Balling R, Gruss P, et al. Waardenburg's syndrome patients have mutations in the human homologue of the Pax-3 paired box gene. Nature. 1992; 355: 635-636.

5. DeStefano AL, Cupples LA, Arnos KS, Asher JH Jr, Baldwin CT, Blanton S, et al. Correlation between Waardenburg syndrome phenotype and genotype in a population of individuals with identified PAX3 mutations. Hum Genet 1998; 102: 449-506. 